\title{
Force Field Analysis Suggests a Lowering of Diffusion Barriers in Atomic Manipulation Due to Presence of STM Tip
}

\author{
Matthias Emmrich, ${ }^{1 *}$ Maximilian Schneiderbauer, ${ }^{1}$ Ferdinand Huber, ${ }^{1}$ Alfred J. Weymouth, \\ Norio Okabayashi, ${ }^{2}$ and Franz J. Giessibl ${ }^{1}$ \\ ${ }^{1}$ Institute of Experimental and Applied Physics, University of Regensburg, D-93053 Regensburg, Germany \\ ${ }^{2}$ Graduate School of Natural Science and Technology, Kanazawa University, Kakuma-machi, Kanazawa, Ishikawa 920-1192, Japan
}

(Received 18 November 2014; revised manuscript received 6 February 2015; published 6 April 2015)

\begin{abstract}
We study the physics of atomic manipulation of $\mathrm{CO}$ on a $\mathrm{Cu}(111)$ surface by combined scanning tunneling microscopy and atomic force microscopy at liquid helium temperatures. In atomic manipulation, an adsorbed atom or molecule is arranged on the surface using the interaction of the adsorbate with substrate and tip. While previous experiments are consistent with a linear superposition model of tip and substrate forces, we find that the force threshold depends on the force field of the tip. Here, we use carbon monoxide front atom identification (COFI) to characterize the tip's force field. Tips that show COFI profiles with an attractive center can manipulate $\mathrm{CO}$ in any direction while tips with a repulsive center can only manipulate in certain directions. The force thresholds are independent of bias voltage in a range from 1 to $10 \mathrm{mV}$ and independent of temperature in a range of 4.5 to $7.5 \mathrm{~K}$.
\end{abstract}

DOI: 10.1103/PhysRevLett.114.146101

PACS numbers: 68.37.Ef, 66.35.+a, 68.37.Ps

In 1959, Richard P. Feynman asked “... whether, ultimately in the great future we can arrange the atoms the way we want; the very atoms, all the way down!" [1]. Thirty years later, that great future was opened by Eigler and Schweizer [2] who arranged Xe atoms on a Ni(110) surface with a low-temperature scanning tunneling microscope. While this technique is used widely now [3-10] — even at room temperature [11] - the physics of atomic manipulation is not fully understood. There have been theoretical investigations [12-17], but experimental studies are scant [18].

When comparing atomic manipulation on a surface to moving a chair around in a room, differences have to be anticipated. The lateral force to move a chair is governed by friction and depends on the normal force-partial compensation of gravity by lifting the chair lowers the force to move it. In 2008, Ternes et al. measured the force needed to move $\mathrm{CO}$ molecules on $\mathrm{Cu}(111)$ and $\mathrm{Co}$ atoms on $\mathrm{Cu}$ and Pt [19]. Unlike moving a chair, they found that the force component normal to the surface did not change the lateral force required to move $\mathrm{CO}$ on $\mathrm{Cu}(111)$. The determined lateral force threshold $F_{\mathrm{thr}}=160 \pm 30 \mathrm{pN}$ is compatible with the force that is needed to move a classical object in the given potential landscape. With the diffusion barrier of $\mathrm{CO} / \mathrm{Cu}(111)$ of $E_{b}=75 \mathrm{meV}[19,20]$ and the atomic spacing for $\mathrm{Cu}(111)$ of $a_{0}=255 \mathrm{pm}$, a sinusoidal model of the surface potential $V(x)=\frac{1}{2} E_{b} \cos \left(2 \pi x / a_{0}\right)$ with a moving threshold of $\left.(d V / d x)\right|_{\max }=148 \mathrm{pN}$ can be deduced-in almost perfect agreement with the measured $160 \pm 30 \mathrm{pN}$ of [19].

In this Letter, we investigate the influence of different tip terminations on lateral manipulation systematically. We use the carbon monoxide front atom identification (COFI) method [21] to identify the tips by their force profile.
The force field is found to determine the mode of manipulation: pulling, pushing, or sliding [3,22]. The first two methods involve a stick-slip motion of the adsorbate, whereas sliding means that it follows the tip smoothly. Depending on the tip, manipulation force thresholds reach $38 \%$ to $51 \%$ of the $150 \mathrm{pN}$ threshold of the classical model. Thus, a linear superposition of the forces acting on the $\mathrm{CO}$ is proven to be invalid and the presence of the tip apparently leads to a lowering of the diffusion barrier in agreement with previous work for manipulation on semiconductors and insulators [11,18,23-25] as well as on metal surfaces $[13,15]$.

Most data presented here were obtained at a temperature of $7.5 \mathrm{~K}$ with a custom-built scanning probe microscope. Force and current were recorded simultaneously by using a qPlus sensor [26]. The sensor was operated in the frequency modulation mode [27] at an amplitude of $50 \mathrm{pm}$ to ensure high sensitivity to short range forces [28]. Etched tungsten tips were prepared by poking them into a clean $\mathrm{Cu}$ sample. The surface was then covered with approximately 0.01 monolayers of $\mathrm{CO}$ molecules. Prior to the $\mathrm{CO}$ manipulation experiments, the orientation of the $\mathrm{Cu}(111)$ lattice was determined by imaging with a $\mathrm{CO}$ functionalized tip [29-31]. Thereafter, the CO was released from the tip. To characterize the force field of the metal tip, threedimensional frequency-shift data $(\Delta f)$ over an adsorbed $\mathrm{CO}$ molecule [21] were acquired and deconvoluted into forces [32]. The COFI images obtained can be classified into two categories [21,33]: attractive center tips (ACTs) and repulsive center tips (RCTs). Recent results show, that ACTs are monoatomic tips, whereas RCTs are multiatomic tips, with the number of attractive dips corresponding to the size of the cluster that forms the tip apex [34]. Ideally, COFI 
images of ACTs feature a repulsive ring with constant height around the attractive minimum. RCTs show two, three, or four attractive dips with similar depth arranged around the repulsive center. In practice, these images are more or less symmetric depending on the tilt angle between the tip axis and the sample normal

The subsequent manipulation experiments were performed in the direction of adjacent top sites, as this manipulation path has the lowest diffusion barrier of $75 \mathrm{meV}[19,20]$. The tip trajectory during manipulation is illustrated in Fig. 1(a). The measurement cycle is stopped at a height $z_{\operatorname{man}}$ where manipulation is observed. At large tip-sample separations, as indicated by faint lines in Figs. 1(b)-1(d), the vertical force profile [Fig. 1(b)] shows (a)

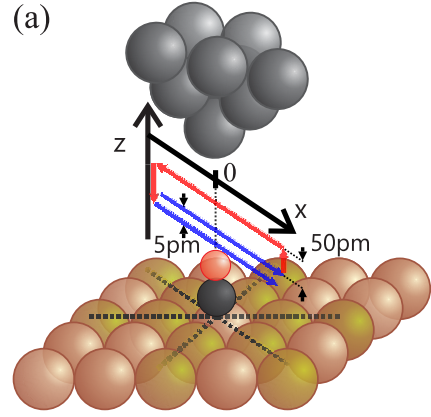

(c)

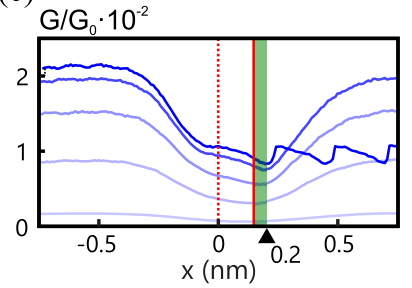

(b)

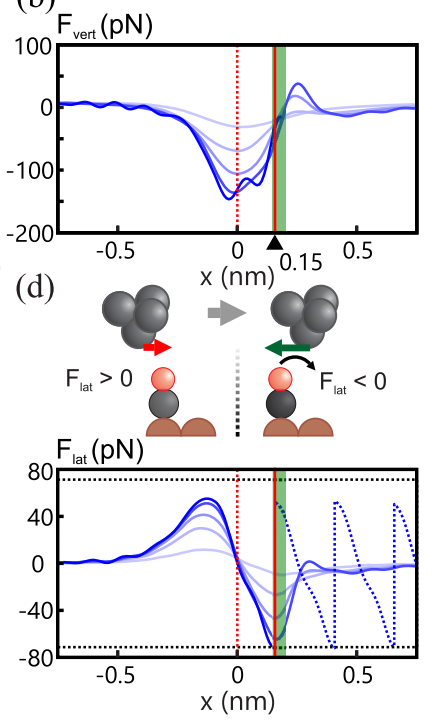

FIG. 1 (color online). (a) An attractive-center tip is scanned along one of the six nearest neighbor directions over a $\mathrm{CO}$ molecule in constant height. Before retrace, the tip is lifted by $50 \mathrm{pm}$ and lowered by $55 \mathrm{pm}$ before commencing the next trace (red arrows) such that each scan cycle lowers the tip by $5 \mathrm{pm}$. (b) Vertical force as a function of the lateral tip position $x$ at different tip heights. Darker lines refer to closer distances. A tilt of the tip gives rise to an asymmetric profile at small $z$ separations. The red dashed line indicates the attractive minimum. (c) Conductance in units of the conductance quantum $G_{0}=2 e^{2} / h$. Because of the tip's tilt, the dip in conductance becomes distorted at close distances and the position of the minimum shifts to the right as marked by the green bar. Steplike features indicate a stick-slip motion of the CO. (d) Lateral forces acting on the tip are obtained by differentiation of the energy in the $x$ direction. For an ACT, lateral forces develop a maximum followed by a minimum where a positive sign indicates that the $\mathrm{CO}$ pulls the tip to the right and vice versa. Because of the slightly tilted tip, the profile of $F_{\text {lat }}$ is asymmetric with extrema of different magnitudes. $F_{\text {lat }}$ needs to exceed the manipulation threshold $F_{\text {thr }}$, indicated by black dashed lines in (d). At the distance shown here, manipulation to the right is feasible. To be able to move $\mathrm{CO}$ to the left, the tip would need to be lowered further such that $F_{\text {lat }}>F_{\text {thr }}$ for negative $x$ values, too. a symmetric attractive minimum over the $\mathrm{CO}$ molecule centered at $x=0 \mathrm{~nm}$ (red dashed line). The averaged normalized conductance $G / G_{0}$ [19] [Fig. 1(c)] exhibits a shallow dip over the $\mathrm{CO}$ in this $z$ regime as expected [29]. For clarity, Figs. 1(b)-1(d) show only a selection of the approximately 100 curves spaced by $\Delta z=5 \mathrm{pm}$; see Fig. S1 in the Supplemental Material [35] for a full set. At smaller separations, the dip becomes more and more distorted due to the tilt of the tip. The conductance minimum does not align with the force minimum. The onset of steps indicates that the $\mathrm{CO}$ is manipulated [22]. For the direction considered here, the manipulation threshold of $\approx 72 \mathrm{pN}$ is overcome by negative lateral forces as shown in Fig. 1(d) (the sign convention used here is explained in the respective figure caption). Manipulation starts when the tip is at $x=0.15 \mathrm{~nm}$, on the right of the $\mathrm{CO}$, indicating a pulling mode.

In the following, the influence of the tip's force field on lateral manipulation is investigated. Examples of an ACT and a RCT are shown by their COFI images in Figs. 2(a) and 2(b). Because of a tilt of the ACT, the repulsive ring is more pronounced on the upper right side. The force versus distance curve $F(z)$ of that tip yields a minimum of $-120 \mathrm{pN}$ [see Fig. S2(b), [35]], identifying it as a $\mathrm{Cu}(100)$ tip [33]. The $F(z)$ curve of the RCT [see Fig. S2(d), [35]] shows quantitative agreement with the $\mathrm{W}(111)$ tips reported in [33]. Possible manipulation paths pointing into the $\langle 1 \overline{1} 0\rangle$ directions of the $\mathrm{Cu}(111)$ surface are indicated in both COFI images. For the ACT, the influence of the tilt is examined by comparing the manipulation behavior in directions $x_{A}$ and $x_{B}$. Results are contrasted to manipulation in direction $x_{C}$, where the force profile is almost perfectly symmetric [Fig. 2(c), force profiles are acquired at a height $\left.z=z_{\operatorname{man}}+5 \mathrm{pm}\right]$. The conductance profile at the manipulation height $z_{\operatorname{man}}$ [Fig. 2(e)] indicates that the $\mathrm{CO}$ has moved in all three experiments. The lateral force curves [Fig. 2(g)] allow for a better understanding of the dynamics of the single processes. In direction $x_{A}$, the minimum and the maximum of the lateral force have different magnitudes $(-62 \mathrm{vs} 48 \mathrm{pN})$. Therefore, the manipulation threshold is reached by negative forces first. As these forces (viewed from the $\mathrm{CO}$ ) point to the right and arise after the tip has passed the $\mathrm{CO}$, pulling occurs. In the reverse direction, the $\mathrm{CO}$ moves due to positive lateral forces directed to the left. According to the conductance profile, it moves one lattice site because the depression is interrupted at $x=-0.25 \mathrm{~nm}$ and the subsequent part is shifted to the left [Fig. 2(e), compare red profile to the red dashed line, showing the conductance $5 \mathrm{pm}$ further away]. For symmetric directions like $x_{C}$, both extrema of the lateral force have the same magnitude ( $55 \mathrm{vs}-56 \mathrm{pN}$ ). Thus, both reach the manipulation threshold at the same tip height. The resulting sliding motion is depicted in Fig. 2(i): On its original position $x=0 \mathrm{pm}$, the CO feels attraction to the left as the tip approaches. The force is sufficient to overcome the barrier. 

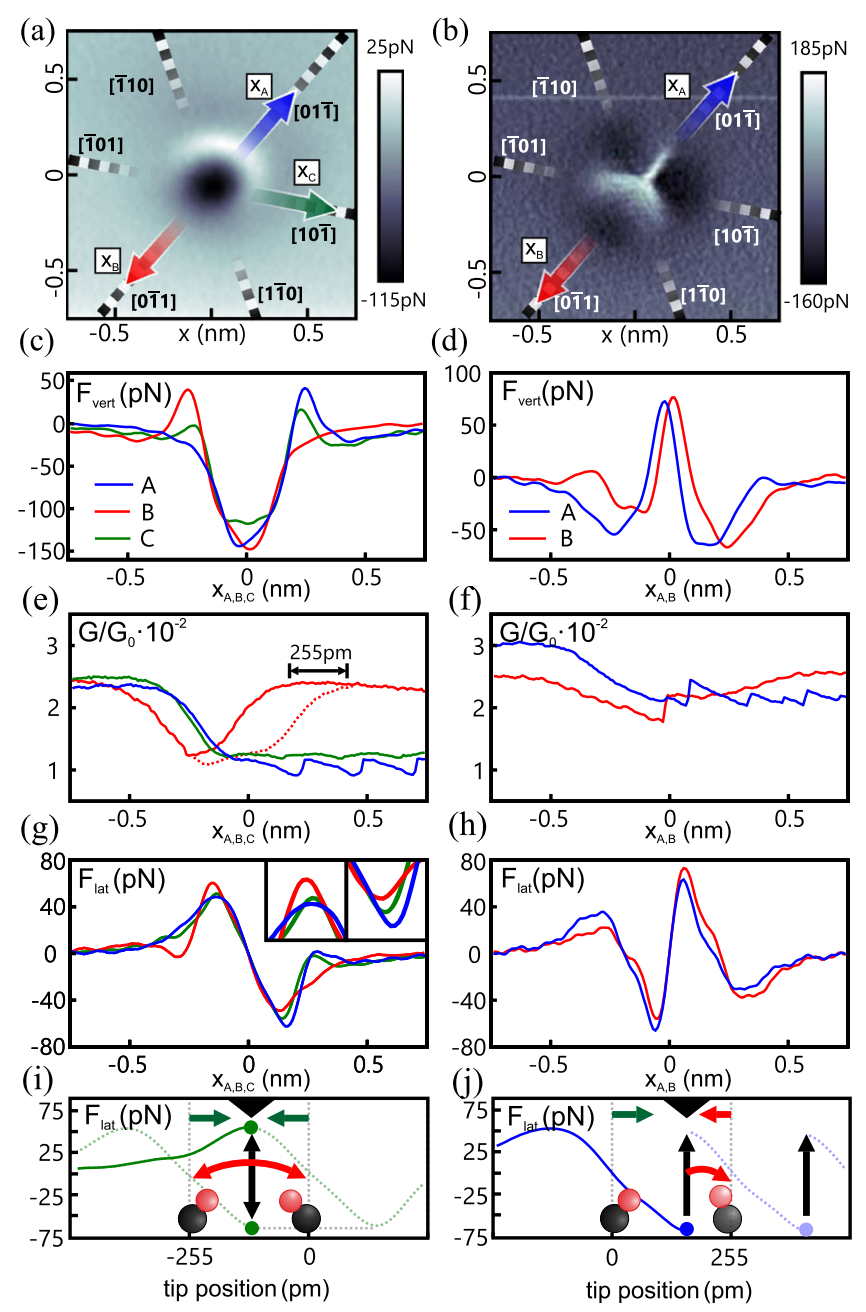

FIG. 2 (color online). Directionality of atomic manipulation for (a) a tilted attractive- and (b) a repulsive-center tip. (c) For the ACT, two asymmetric directions $x_{A}$ and $x_{B}$, and one almost symmetric direction $x_{C}$ are selected (profiles taken at $z_{\operatorname{man}}+5 \mathrm{pm}$ ). (d) For the RCT, manipulation with the sickle shaped part (direction $x_{A}$ ) is compared to the case where the beamlike feature comes first (direction $x_{B}$ ). The difference in the profiles results from the $\mathrm{CO}$ bending to the side. Evidence of the $\mathrm{CO}$ motion is found in the average normalized conductance $G / G_{0}$ at $z_{\text {man }}$ : (e) For the ACT manipulation is observed in directions $x_{A}$ and $x_{C}$. The shape of the curve in direction $x_{B}$ indicates that the $\mathrm{CO}$ has moved to the left. (f) Manipulation with a RCT is only possible in direction $x_{A}$. When the beam hits the CO first, it is pushed to the side. (g) The lateral force curves acquired with the ACT reflect its symmetry properties (see insets). In direction $x_{B}$ the threshold is reached by positive forces explaining the motion of the $\mathrm{CO}$ to the left. (h) Repulsive lateral forces point away from the molecule. Regardless of the slight asymmetry the $\mathrm{CO}$ is always moved when the tip is left of the CO. (i) During sliding, the CO is forced under the tip by lateral forces of different sign but equal strength. (j) Tilted ACTs that favor forces pointing in the direction of the tip motion pull the $\mathrm{CO}$.

On the temporary adsorption site at $x=-255 \mathrm{pm}$ the respective lateral force points into the opposite direction and is again large enough for manipulation. At this point, neither site is favored. The CO is trapped and directionality is induced by the tip motion. Our explanation of sliding is different from Ref. [38], where sliding was supposed to set in approximately $10 \mathrm{pm}$ closer than the threshold for pulling due to the tip potential being larger than the diffusion barrier. We only observe pulling for tilted tips that favor attractive lateral forces pointing into the direction of tip motion as schematically shown in Fig. 2(j). If such a tip is approached closer, forces pointing in the opposite direction also reach the threshold force and the manipulation mode changes from pulling to sliding.

The RCT in Fig. 2(b) features a very sharp repulsive center, surrounded by three attractive lobes that are separated by repulsive beams. This particular RCT was chosen because the beams align nicely with the $\langle\overline{1} 10\rangle$ directions of the substrate. For this tip, we investigate manipulation in direction $x_{A}$, where a repulsive sickle with two beams in the $[\overline{1} 01]$ and $[1 \overline{1} 0]$ directions makes contact with the CO first. We compare this to the reverse direction $x_{B}$. Although the attractive lobes can induce forces of the same magnitude as the ACTs, repulsion dominates the vertical force profile shown in Fig. 2(d). The CO is not stable on the repulsive beam facing the $[01 \overline{1}]$ direction; thus, attractive minima can be found on both sides of the repulsive peak. According to the conductance channel [Fig. 2(f)] continuous manipulation is only possible in direction $x_{A}$. In direction $x_{B}$, a discontinuous increase of the conductance can be seen at $x=0$. This indicates that the CO has moved sideways out of the desired manipulation path because it is not stable in front of the repulsive beam. The sequence of minimum and maximum in the lateral force curve of the RCT [Fig. 2(h)] is reversed to the one of the ACT. This is caused by the sharpness of the center feature of the RCT, making repulsion the prevailing feature of the lateral force profile. In both directions $x_{A}$ and $x_{B}$, the $\mathrm{CO}$ moves due to negative lateral forces arising at tip positions left of the center of the molecule. Thus, pushing is the only manipulation mode observed for RCTs and only possible for directions where the molecule is guided between two repulsive beams.

All lateral forces reported in this article are smaller than the forces in [19] and what would be expected from the diffusion barrier model introduced earlier in the text.

Because forces are extracted from the profiles taken at $z_{\text {man }}+5 \mathrm{pm}$, their values are slightly underestimated. In Fig. S3(a) of the Supplemental Material [35] the logarithm of the maximum lateral force is plotted as a function of the tip height $z$. Extrapolation of the fit to $z_{\operatorname{man}}$ yields an upper boundary for the force threshold roughly $10 \%$ higher than the listed values. However, the diffusion barrier model and the measurements in [19] indicate at least twice the threshold values stated here. To track down the physics behind the low threshold forces, manipulation experiments with many different tips have been performed. Most tip terminations used in the manipulation experiments can be identified by their specific $F(z)$ curves, taken over the center of the $\mathrm{CO}$ [33]. Figure 3(a) relates the force threshold 

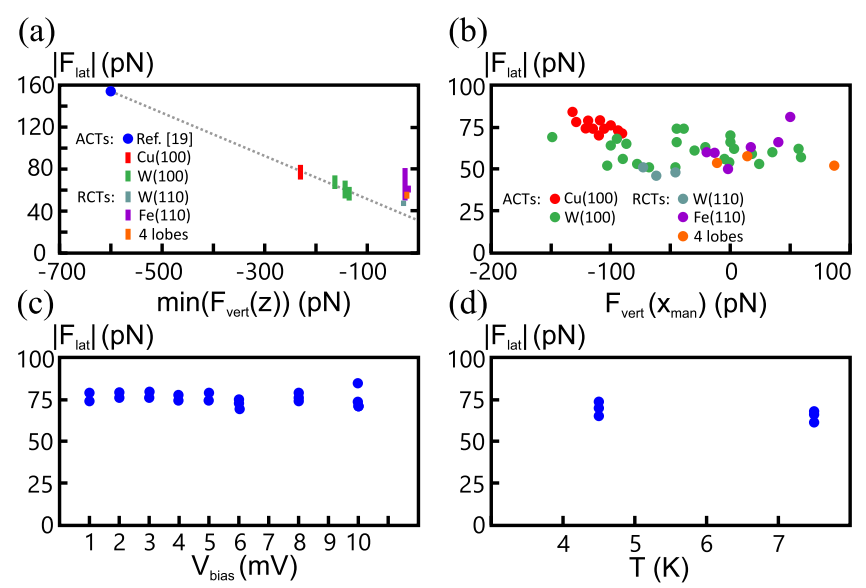

FIG. 3 (color online). (a) Manipulation threshold at $z_{\operatorname{man}}+$ $5 \mathrm{pm}$ as a function of the maximal attractive normal force determined from $F(z)$ for several tips. Tip terminations are assigned according to [33]. Apparently, the manipulation threshold increases with the maximal attraction a tip can generate. (b) The manipulation threshold $F_{\text {thr }}$ does not depend on the normal forces. (c) $F_{\text {thr }}$ as a function of bias voltage, showing no apparent correlation for voltages between 1 and $10 \mathrm{mV}$. (d) The manipulation threshold has a weak temperature dependence. At $7.5 \mathrm{~K}$ it is $5 \mathrm{pN}$ lower than at $4.5 \mathrm{~K}$.

to the tip termination, represented by the characteristic depth of the attractive force minimum of the $F(z)$ curve. For ACTs we find that tips which develop more attraction over the $\mathrm{CO}$ require more force to move the molecule. Extrapolating this trend to vertical forces of $-600 \mathrm{pN}$ yields lateral forces around $150 \mathrm{pN}$-close to the values reported in [19]. These results suggest the lateral force exerted by the tip on the CO molecule is not the only driver for motion, but the presence of the tip apparently lowers the diffusion barrier, as predicted $[13,15]$. We confirm this prediction and find that the threshold force to move $\mathrm{CO} / \mathrm{Cu}(111)$ with ACTs depends on the atomic species: For $\mathrm{Cu}(100)$ tips $61 \pm 6 \mathrm{pN}$ are needed, compared to $76 \pm 2 \mathrm{pN}$ for $\mathrm{W}(100)$ and $160 \pm 30 \mathrm{pN}$ for the ACT Ir tip in [19]. RCTs yield an average of $57 \pm 7 \mathrm{pN}$ independent of the atomic composition. The force threshold for moving $\mathrm{CO}$ with an Ir tip was found to be independent of the vertical forces in Ref. [19]. Manipulation with the $\mathrm{Cu}$, $\mathrm{W}$, and Fe tips considered here verifies that observation: The plot of lateral versus vertical forces [Fig. 3(b)] measured at the tip position $x_{\text {man }}$ where the $\mathrm{CO}$ gets manipulated shows fluctuating values of $F_{\text {thr }}$ but no correlation with vertical forces. The frustrated translational mode of $\mathrm{CO} / \mathrm{Cu}(111)$ has an energy of $4 \mathrm{meV}$. For bias voltages above $4 \mathrm{mV}$ this mode could be excited by inelastic tunneling processes, which would enable the $\mathrm{CO}$ to overcome the barrier at lower force thresholds $[10,39,40]$. Recurring manipulation in a fixed direction using voltages between 1 and $10 \mathrm{mV}$ was conducted. As shown in Fig. 3(c), force thresholds spread around
$76 \pm 2 \mathrm{pN}$ but do not correlate with bias. The diffusion rate of adsorbates on the surface follows an Arrhenius law. For an assembly of three COs in the metastable "chevron" configuration, the exponential increase with $T$ starts to emerge at $6.5 \mathrm{~K}$ [41]. Ternes et al. observed the $160 \mathrm{pN}$ force threshold at a temperature of $5 \mathrm{~K}$. As our instrument was at $7.5 \mathrm{~K}$, the low values for the force threshold could be a result of thermal excitation. Control experiments on a different microscope at nominal temperatures of 4.5 and $7.5 \mathrm{~K}$ with the same tip [Fig. 3(d)] show no significant change of the threshold force in this temperature range.

In summary, we have shown that the termination of the tip determines the lateral manipulation force threshold of an adsorbate on a metal surface, a finding that will also be relevant for autonomous atom assemblers [42]. Apparently, the presence of the tip lowers the diffusion barrier and thus the threshold force from the value given by a lateral derivative of the surface potential. Radially symmetric tips that induce high attractive forces over the $\mathrm{CO}$ require larger force thresholds. Apparently, the presence of the tip lowers the diffusion barrier and thus the threshold force from the value given by a lateral derivative of the surface potential. Radially symmetric tips that induce high attractive forces over the $\mathrm{CO}$ require larger force thresholds. The symmetry of the tip's force field defines the mode of manipulation. For ACTs, the manipulation mode is determined by the tilt of the tip. RCTs can only push in directions when the adsorbate is stabilized by features of the tip.

The authors thank J. Repp and L. Gross for discussions and the Deutsche Forschungsgemeinschaft for funding within the research Project No. SFB 689.

*matthias.emmrich@ur.de

[1] R. P. Feynman, There's Plenty of Room at the Bottom, Caltech (1959), http://www.zyvex.com/nanotech/feynman .html.

[2] D. M. Eigler and E. K. Schweizer, Nature (London) 344, 524 (1990).

[3] J. A. Stroscio and D. M. Eigler, Science 254, 1319 (1991).

[4] P. Zeppenfeld, C. Lutz, and D. Eigler, Ultramicroscopy 42-44, 128 (1992).

[5] M. F. Crommie, C. P. Lutz, and D. M. Eigler, Science 262, 218 (1993).

[6] G. Meyer and K.-H. Rieder, Surf. Sci. 377-379, 1087 (1997).

[7] A. A. Khajetoorians, J. Wiebe, B. Chilian, and R. Wiesendanger, Science 332, 1062 (2011).

[8] S. Loth, S. Baumann, C. P. Lutz, D. M. Eigler, and A. J. Heinrich, Science 335, 196 (2012).

[9] K. K. Gomes, W. Mar, W. Ko, F. Guinea, and H. C. Manoharan, Nature (London) 483, 306 (2012).

[10] A. A. Khajetoorians, B. Baxevanis, C. Hübner, T. Schlenk, S. Krause, T. O. Wehling, S. Lounis, A. Lichtenstein, D. Pfannkuche, J. Wiebe, and R. Wiesendanger, Science 339, 55 (2013). 
[11] Y. Sugimoto, M. Abe, S. Hirayama, N. Oyabu, O. Custance, and S. Morita, Nat. Mater. 4, 156 (2005).

[12] X. Bouju, C. Girard, H. Tang, C. Joachim, and L. Pizzagalli, Phys. Rev. B 55, 16498 (1997).

[13] C. Ghosh, A. Kara, and T. S. Rahman, Surf. Sci. 502-503, 519 (2002).

[14] L. Pizzagalli and A. Baratoff, Phys. Rev. B 68, 115427 (2003).

[15] C. Ghosh, A. Kara, and T.S. Rahman, J. Nanosci. Nanotechnol. 6, 1068 (2006).

[16] Y. Xie, Q. Liu, P. Zhang, W. Zhang, S. Wang, M. Zhuang, Y. Li, F. Gan, and J. Zhuang, Nanotechnology 19, 335710 (2008).

[17] Y.-Q. Xie, T.-X. Yang, X. Ye, and L. Huang, Appl. Surf. Sci. 258, 1139 (2011).

[18] Y. Sugimoto, P. Jelinek, P. Pou, M. Abe, S. Morita, R. Perez, and O. Custance, Phys. Rev. Lett. 98, 106104 (2007).

[19] M. Ternes, C. P. Lutz, C. F. Hirjibehedin, F. J. Giessibl, and A. J. Heinrich, Science 319, 1066 (2008).

[20] K. L. Wong, B. V. Rao, G. Pawin, E. Ulin-Avila, and L. Bartels, J. Chem. Phys. 123, 201102 (2005).

[21] J. Welker and F. J. Giessibl, Science 336, 444 (2012).

[22] L. Bartels, G. Meyer, and K.-H. Rieder, Phys. Rev. Lett. 79, 697 (1997).

[23] M. B. Watkins and A. L. Shluger, Phys. Rev. B 73, 245435 (2006).

[24] T. Trevethan, L. Kantorovich, J. Polesel-Maris, S. Gauthier, and A. Shluger, Phys. Rev. B 76, 085414 (2007).

[25] Y. Sugimoto, K. Miki, M. Abe, and S. Morita, Phys. Rev. B 78, 205305 (2008).

[26] F. J. Giessibl, Appl. Phys. Lett. 73, 3956 (1998).
[27] T. R. Albrecht, P. Grütter, D. Horne, and D. Rugar, J. Appl. Phys. 69, 668 (1991).

[28] F. J. Giessibl, H. Bielefeldt, S. Hembacher, and J. Mannhart, Appl. Surf. Sci. 140, 352 (1999).

[29] L. Bartels, G. Meyer, and K.-H. Rieder, Appl. Phys. Lett. 71, 213 (1997).

[30] M. Böhringer, W.-D. Schneider, and R. Berndt, Surf. Sci. 408, 72 (1998).

[31] J. A. Stroscio and R. J. Celotta, Science 306, 242 (2004).

[32] J. E. Sader and S. P. Jarvis, Appl. Phys. Lett. 84, 1801 (2004).

[33] T. Hofmann, F. Pielmeier, and F. J. Giessibl, Phys. Rev. Lett. 112, 066101 (2014).

[34] M. Emmrich et al., Science (2015).

[35] See Supplemental Material at http://link.aps.org/ supplemental/10.1103/PhysRevLett.114.146101 for a description of the experimental methods, an evaluation of the accuracy of the stated values and a detailed discussion of the barrier lowering, which includes Refs. [36,37].

[36] F. J. Giessibl, Rev. Mod. Phys. 75, 949 (2003).

[37] N. Knorr, H. Brune, M. Epple, A. Hirstein, M. A. Schneider, and K. Kern, Phys. Rev. B 65, 115420 (2002).

[38] A. Kühnle, G. Meyer, S. Hla, and K.-H. Rieder, Surf. Sci. 499, 15 (2002).

[39] I. Bat'ko and M. Bat'ková, Eur. Phys. J. Appl. Phys. 31, 191 (2005).

[40] S. Krause, L. Berbil-Bautista, G. Herzog, M. Bode, and R. Wiesendanger, Science 317, 1537 (2007).

[41] A. J. Heinrich, C. P. Lutz, J. A. Gupta, and D. M. Eigler, Science 298, 1381 (2002).

[42] R. J. Celotta, S. B. Balakirsky, A. P. Fein, F. M. Hess, G. M. Rutter, J. A. Stroscio, Rev. Sci. Instrum. 85, 121301 (2014). 